\title{
Evaluation and prospects from a measurement campaign on real multimedia traffic in LTE vs. UMTS
}

Scott Fowler, Jalal Sarfraz, M. Muddassir Abbas, Erik Bergfeldt and Vangelis Angelakis

\author{
Linköping University Post Print
}

\section{Tweet}

N.B.: When citing this work, cite the original article.

Original Publication:

Scott Fowler, Jalal Sarfraz, M. Muddassir Abbas, Erik Bergfeldt and Vangelis Angelakis, Evaluation and prospects from a measurement campaign on real multimedia traffic in LTE vs. UMTS, 2014, Wireless Communications, Vehicular Technology, Information Theory and Aerospace Electronic Systems (VITAE'14), IEEE 4th International Conference, 1-5.

http://dx.doi.org/10.1109/VITAE.2014.6934475

(C2014 IEEE. Personal use of this material is permitted. However, permission to reprint/republish this material for advertising or promotional purposes or for creating new collective works for resale or redistribution to servers or lists, or to reuse any copyrighted component of this work in other works must be obtained from the IEEE.

http://ieeexplore.ieee.org/

Postprint available at: Linköping University Electronic Press http://urn.kb.se/resolve?urn=urn:nbn:se:liu:diva-112789 


\title{
Evaluation and Prospects from a Measurement Campaign on Real Multimedia Traffic in LTE vs. UMTS
}

\author{
Scott Fowler, Jalal Sarfraz, Muhammad Muddassir Abbas, Erik Bergfeldt, and Vangelis Angelakis \\ Department of Science \& Technology, Linköping University, Sweden
}

\begin{abstract}
The LTE standard has been developed for highbandwidth mobile access for today's data-heavy applications. With broadband cellular available already from the UMTS system, users are expecting the LTE network to provide high quality communication. To ensure the quality and correctness of complex systems of LTE, it is important to evaluate the behavior of traffic on a LTE network in relation to UMTS. In this paper, LTE and UMTS measurements on video traffic were performed in a real environment, allowing for greater development, and understanding of network traffic performance.
\end{abstract}

\section{INTRODUCTION}

Recent advancements in telecommunication systems have created a new trend in the consumer electronics market. A large variety of smart mobile devices (e.g. iPhone, iPad, Android, Windows) powerful enough to support a wide range of multimedia traffic [1]-[3] have now become a ubiquitous commodity. Thus, mobile data traffic is expected to increase dramatically by 2018 [4]. According to this forecast, Cisco Systems is predicting that video traffic will be $69 \%$ of the internet traffic by 2018 resulting in greater bandwidth usage on the limited wireless channels. However, limitations in speed and latency have made $3 \mathrm{G}$ services unsuitable for high-end multimedia traffic, especially in dense environments.

Thus, the increasing demand of high speed data services; 3rd Generation Partnership Project (3GPP) introduced the new radio access technology, LTE (Long Term Evolution) and LTEAdvanced which has the capability to provide larger bandwidth and low latencies on a wireless network in order to fulfill the demand of Users' Equipment (UEs) with acceptable Quality of Service (QoS).

Even though already there is a significant global rollout of LTE, there is little known about the usage behavior and the traffic characteristics of the LTE mobile devices. By knowing how the profile of a LTE mobile device differ from Universal Mobile Telecommunications System (UMTS) mobile device, a manufacturer would be able to use this information in the design of resource allocation algorithms in base stations and other mobile device. By understanding about the distribution size of objects transferred, one would be able to minimize protocol overhead and adapt holding times for design power saving on mobile devices battery [5]-[7]. For future wireless networks, it is crucial to identify traffic characteristics in order to improve or design new PHY and MAC layer techniques.
However, despite its high potential, LTE is still at its early stages. Thus, a major ground work has to be done to identify areas which require improving communication quality. Traffic measurements are effective means to determine how well a protocol or an application is performing in the wireless environment. Utilizing this detailed analysis of network measurement will identify performance bottlenecks of LTE. Once these performance problems are addressed, the new versions of protocols can enable better performance for the end users.

Although there have been mathematical models for LTE to study identify performance bottlenecks, they lack the hard reality of real conditions. In this paper we present an empirical measurement study of LTE and UMTS network traffic using real LTE equipment with daily life applications: Skype video calls and real-time video (live web) streaming. We measured the inter-arrival time of packets to characterize network behaviors of LTE and UMTS using a USB modem capable of $3 \mathrm{G}$ and $4 \mathrm{G}$.

\section{TESTING ENVIRONMENT}

The data collection was performed with the Samsung GT-B3730 USB dongle modem. The modem is capable of supporting LTE $(2600 \mathrm{MHz}), 3 \mathrm{G}$ HSPA/UMTS $(2100 \mathrm{MHz})$, and $2 \mathrm{G} / \mathrm{EDGE}(900 / 1800 \mathrm{MHz})$ with the peak uplink/downling speeds of $100 / 50 \mathrm{Mbps}$ for LTE, $17 / 5.76 \mathrm{Mbps}$ for HSPA/UMTS, and 296/107 Kbps for 2G/EDGE. The device came with its own drivers for Windows, Linux, and Mac operating systems.

We collected the measurements for both LTE and UMTS by connecting the device to a desktop computer with an Intel Core 2 Duo $1.6 \mathrm{GHz}$ processor and 1GB RAM. The Wireshark Network Protocol Analyzer v. 1.6.7 was used to capture network traces for Skype video calls and live web streaming. Over a period of 3 months a large amount of measurements was collected: approximately 1 million packets per traffic type, allowing for a sufficient amount of data to produce meaningful statistical results and conclusions.

\section{A. Traffic Analysis Methodologies}

All the raw data and network traces were processed to enable maximum information retrieval. Source and the destination IPs were identified along with their port numbers to visualize the traffic flow between them. This data was further re-arranged in a manner to calculate the Inter-arrival Time (IATs). In traffic 
modelling and analysis, the packet sizes and the IAT between the packets are the two commonly used parameters. In our paper, all the statistical calculations revolve around the IATs of the measured traffic streams.

To obtain the general trend of the data collected, nonparametric density estimation was applied to convert the discretised IATs into continuous time data. Typically, such nonparametric methods assume that the data has been taken from no particular probability distribution, opposed to the parametric methods which assume that the data follows a particular probability distribution and consequently the parameters of the distribution. Thus, our technique assumes no fixed structure on the model. Due to the minuscule assumptions and the simplicity these methods are more widely applicable than parametric methods.

\section{SKYPE VideO CALL ANALYSis}

Skype is one of the most widely used VoIP applications these days. Skype's high quality voice and video, and the ability to maintain connection with minimum interference has made it popular among users as compared to its competitors.

Skype does not operate based on a typical client-server based model but all calls are encrypted and it has a peer-to-peer (P2P) structure similar to the popular file sharing application KaZaa [8]

For Skype, v. 5.10.0.114 was installed on the desktop data collector. The calling party was using an intel Core i3 based laptop with 2GB RAM running the same Skype version. The observations for the applications were gathered by running them one by one, and all other data transfer programs were stopped during the data collection.

Skype stores user information in a decentralized manner [9] and there are no central services in Skype except for services like authentication, contacting user list storage and connecting to PSTN.

\section{A. Results for Skype Video Call}

In this section we will discuss the results for Skype Video call for LTE and UMTS. Wireshark was used to capture a large sample of measurements.

Figure 1 shows the Cumulative Distribution Function (CDF) of the Inter-arrive Times (IATs) for LTE and UMTS video call. If we see the individual CDF for LTE we can observe that from around $0.05 \mathrm{~ms}$ to $1 \mathrm{~ms}$ constitutes about $40 \%$ and from $1 \mathrm{~ms}$ to around $13 \mathrm{~ms}$ constitutes the next $50 \%$ and above $13 \mathrm{~ms}$ constitutes the rest of the $10 \%$ IATs. It means that $90 \%$ of the IATs for Video Call lie below 13ms.

Similarly, when we see the individual CDF of UMTS we observe that from around $0.03 \mathrm{~ms}$ to $1 \mathrm{~ms}$ constitute about $40 \%$ of the IATs and from $1 \mathrm{~ms}$ to $10 \mathrm{~ms}$ are the next $30 \%$ while from $10 \mathrm{~ms}$ to around $37 \mathrm{~ms}$ are the next of the $25 \%$ while IATs above $37 \mathrm{~ms}$ are the rest of the $5 \%$ of the total. The spread of the IATs of video call for both LTE and UMTS can be viewed by the percentiles given in Table I.

When we compare the CDFs for LTE and UMTS, we see that in the first $40 \%$ part, the IATs of the UMTS are less than that of LTE. But as we move from 1ms onwards, we observe

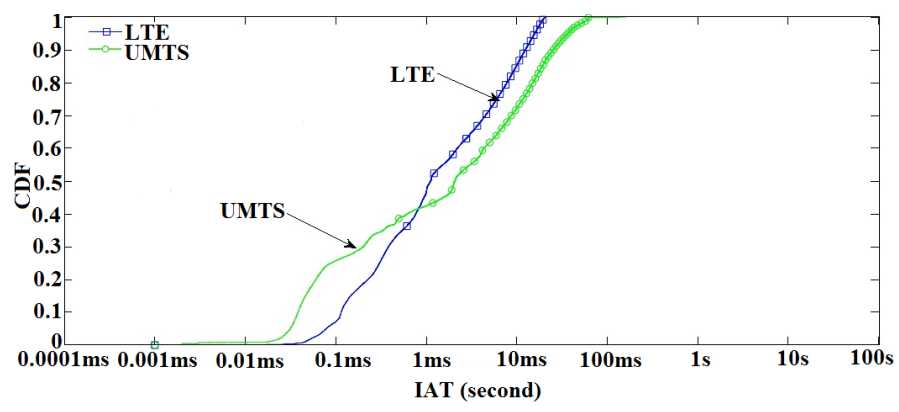

Figure 1: CDF of the IATs for LTE and UMTS Video Call

that LTE is faster than UMTS as its IATs are less than that of UMTS. Thus, from Figure 1 it evident that most of the time (about $60 \%$ ) LTE was faster than UMTS. Also, with the aid of Table I, we can see that almost $68 \%$ of the IATs of LTE lies below the mean value of $3.9 \mathrm{~ms}$ with a standard deviation of $5.4 \mathrm{~ms}$; for UMTS, although almost $70 \%$ of its IATs lies below the mean value of $8.8 \mathrm{~ms}$ as well but the variation is very high as shown by the standard deviation $35.8 \mathrm{~ms}$ which advocates for the stability of the LTE network.

Figure 2 shows the CDF of packet sizes of video call for both LTE and UMTS. When we see the individual CDF of packet sizes for LTE, it is observed that packet sizes from 39-166 Bytes covers about 58\% of the total. From 166-1313 Bytes the ratio is about 7.5\%. From 1313-1386 Bytes the ratio is about $7.5 \%$, and from 1386 Bytes onward till 1434 Bytes about $27 \%$ packet sizes fall in this category.

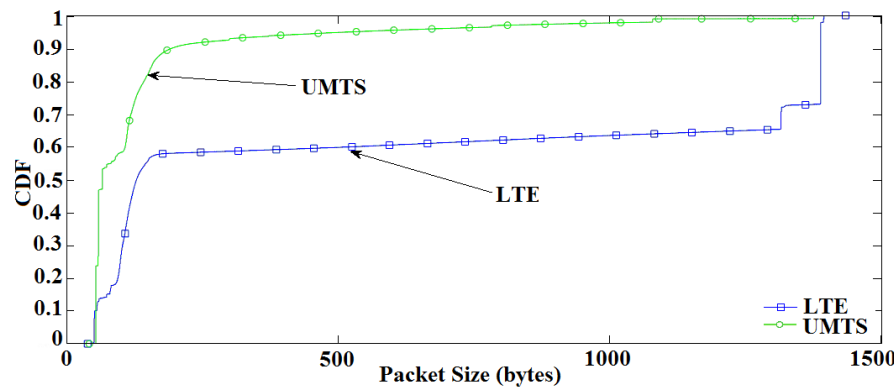

Figure 2: CDF of Packet Sizes for LTE and UMTS Video Call

Similarly for UMTS, packet sizes from 53-66 Bytes covers about $53 \%$ of the total. From 66-120 Bytes the ratio is about $17 \%$. From 120-185 Bytes the ratio is about 20\%, and from 185 Bytes onward are only about $10 \%$ of the total packet sizes.

When we compare the two wireless communication standards in Figure 2, we can clearly see that for most part of the communication, UMTS uses much smaller packets as compared to LTE. Specifically, about $90 \%$ of the packet sizes for UMTS fall below 185 Bytes. This can also be verified from Table II, where the five most frequent packets for both the networks are listed. It can be seen that the most frequent 
packet size for LTE is 1387 Bytes while that of UMTS is only of 54 Bytes. Also, the next two most frequent packets for LTE are of 51 and 1314 Bytes while that of UMTS are of only 59 and 66 Bytes.

Table I: Statistics of Video Call for LTE \& UMTS IAT

\begin{tabular}{|c|c|c|c|c|c|}
\hline \multicolumn{5}{|c|}{ Statistics of IATs for LTE Video Call } \\
\hline$\langle$ IATs $\rangle(\mathrm{ms})$ & \multicolumn{5}{|c|}{3.9} \\
\hline$\sigma$ of IATs (ms) & \multicolumn{5}{|c|}{5.4} \\
\hline Percentiles & $5 \%$ & $25 \%$ & $50 \%$ & $75 \%$ & $95 \%$ \\
\hline $\log ($ IAT) & -4.10 & -3.52 & -2.95 & -2.22 & -1.80 \\
\hline duration & 78 us & $303 \mathrm{us}$ & $1.1 \mathrm{~ms}$ & $6.0 \mathrm{~ms}$ & $15.7 \mathrm{~ms}$ \\
\hline \multicolumn{6}{|c|}{ Statistics of IATs for UMTS Video Call } \\
\hline$\langle$ IATs $\rangle(\mathrm{ms})$ & \multicolumn{5}{|c|}{3.8} \\
\hline$\sigma$ of IATs (ms) & \multicolumn{5}{|c|}{35.8} \\
\hline Percentiles & $5 \%$ & $25 \%$ & $50 \%$ & $75 \%$ & $95 \%$ \\
\hline $\log ($ IAT) & -4.51 & -4.05 & -2.69 & -1.93 & -1.44 \\
\hline duration & $31 \mathrm{us}$ & $89 \mathrm{us}$ & $2.1 \mathrm{~ms}$ & $11.7 \mathrm{~ms}$ & $36.7 \mathrm{~ms}$ \\
\hline
\end{tabular}

Table II: Statistics of Video Call for LTE \& UMTS Packets

\begin{tabular}{|c|c|c|}
\hline \multicolumn{3}{|c|}{ Statistics of Packet Sizes for LTE Skype Video Call } \\
\hline Total Packets & & 885014 \\
\hline Average Packets/s & & 257.5 \\
\hline Average throughput & & $1.21 \mathrm{Mbps}$ \\
\hline Average Packet size (Bytes) & & 587.71 \\
\hline \multicolumn{3}{|c|}{ Five Most Frequent Packet Sizes } \\
\hline Packet Sizes (Bytes) & Instances & Relative Frequency (\%) \\
\hline 1387 & 219377 & 24.79 \\
\hline 51 & 65923 & 7.45 \\
\hline 1314 & 46850 & 5.29 \\
\hline 52 & 19147 & 2.16 \\
\hline 56 & 13671 & 1.54 \\
\hline \multicolumn{3}{|c|}{ Statistics of Packet Sizes for UMTS Video Call } \\
\hline Total Packets & & 939268 \\
\hline Average Packets/s & & 114.12 \\
\hline Average rate & & $0.128 \mathrm{Mbps}$ \\
\hline Average Packet size (Bytes) & & 140.14 \\
\hline \multicolumn{3}{|c|}{ Five Most Frequent Packet Sizes } \\
\hline Packet Sizes (Bytes) & Instances & Relative Frequency (\%) \\
\hline 54 & 222778 & 23.72 \\
\hline 59 & 188624 & 20.08 \\
\hline 66 & 57459 & 6.12 \\
\hline 58 & 27220 & 2.90 \\
\hline 111 & 12075 & 1.29 \\
\hline
\end{tabular}

Furthermore, the average packet size of LTE; 588 Bytes as well as the average packets per second 258 packets are much greater than the average packet size of UMTS; 140 Bytes and the average packets per second 114 packets. Therefore, the average bits per second of LTE; 1.21 Mbps is also much greater than that of UMTS; $0.128 \mathrm{Mbps}$. The detailed packet details are presented in Table II. Thus, LTE is not only faster but also more stable than the UMTS network, and performs much better for video call.

\section{Live Streaming ANALYSis}

There presently two types of dominating streaming services [10]: Video on Demand (VoD) and Live Video Streaming (LVS). In VoD, the user has the control to pause, rewind, forward, and re-play [11] the video, just as local video file. LVS is stripped of such controlling functionalities as videos are directly streaming from the broadcasting servers. Having specific software and hardware capabilities, these servers play a very important role in delivering the required contents to the user. Moreover, these servers have the ability to manage large traffic loads and to select video dynamically on the basis of channel bandwidth [12]. In [13] described the system architecture of a conventional server in more detail.

LVS has two important Quality of Service (QoS) parameters namely playback delay and playback smooth [14], in order for the end user to be able to experience fluent streaming with negligible delay. Hence, it has become a challenging task for the evolving networks to provide higher bandwidth so as have a stable streaming throughput. Common protocols which are used in video streaming are Real Time Streaming Protocol (RTSP), Real Time Protocol (RTP), and Session Description Protocol (SDP) which are discussed in [15].

\section{A. Results for Live Streaming}

In this section we will be discussing the results for LVS for LTE and UMTS. A live football match was played on the computer for each of the networks and the data was recorded via Wireshark. We captured more than 1 million packets, as described previously, to have a sufficient amount of data to produce reasonable results.

Figure 3 shows the CDF of the IATs for LTE and UMTS live streaming. If we see the individual CDF for LTE we can observe that about $60 \%$ of the IATs for LTE live streaming do not go beyond $0.01 \mathrm{~ms}$, the next $20 \%$ lies within $0.01 \mathrm{~ms}$ and $0.1 \mathrm{~ms}$, the next $15 \%$ ranges from $0.1 \mathrm{~ms}$ to around $44 \mathrm{~ms}$, and the rest of the $5 \%$ goes beyond $100 \mathrm{~ms}$. It means that $95 \%$ of the IATs for LTE live streaming do not go beyond $44 \mathrm{~ms}$.

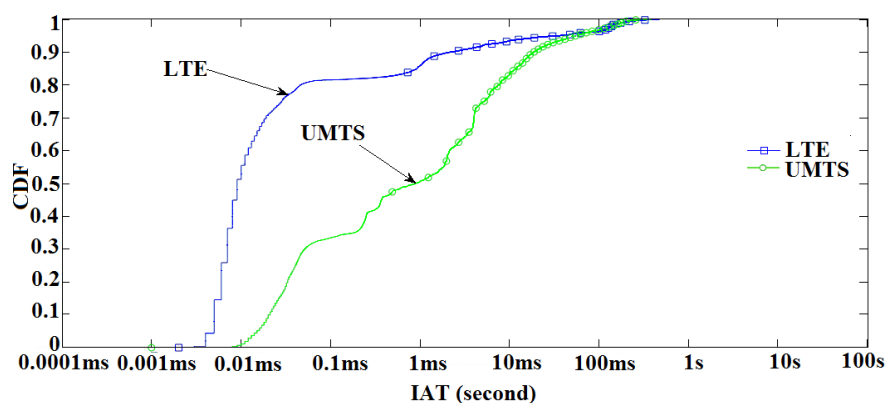

Figure 3: CDF of the IATs for LTE and UMTS Live Streaming

Similarly, when we see the individual CDF of UMTS we observe about $33 \%$ of the data lies at or below $0.1 \mathrm{~ms}$, next $17 \%$ lies between $0.1 \mathrm{~ms}$ to $1 \mathrm{~ms}$, the following $33 \%$ of the total are between $1 \mathrm{~ms}$ to $10 \mathrm{~ms}$; the subsequent $12 \%$ starts from around 
$10 \mathrm{~ms}$ and goes up to $58.2 \mathrm{~ms}$, and the rest of IATs starts from $58.2 \mathrm{~ms}$ and goes beyond $100 \mathrm{~ms}$. The spread of the IATs of live streaming for both LTE and UMTS can be viewed by the percentiles given in Table III.

When we compare the two wireless communication standards, we can clearly see that the IATs of LTE streaming are much smaller than that of UMTS and virtually there is no significant part in the CDF where IATs of UMTS are smaller than that of LTE. Since the IATs of the LTE are smaller, its mean $8.5 \mathrm{~ms}$ (Table III) is also smaller than the mean of the IATs of UMTS, $11.3 \mathrm{~ms}$. Moreover, almost $93 \%$ of the LTE IATs fall less than the mean value indicating that only $7 \%$ of the LTE IATs are greater than $8.5 \mathrm{~ms}$, but for UMTS, $84 \%$ of the IATs are less than the mean value $11.3 \mathrm{~ms}$, meaning $16 \%$ of the IATs are greater than $11.3 \mathrm{~ms}$. Thus, the packets of the LTE arrived much faster than the UMTS in live streaming.

When we see the standard deviation of both the networks as given in Table III, there is not much of a difference but the standard deviation of UMTS is a little higher when compared to its mean. Although, the difference is not much LTE also performs better when it comes to variation among the IATs which can affect the latency and jitter in the video as it plays an important role in live streaming and one wants to have as smooth a stream as possible.

Figure 4 shows the CDF of packets sizes for LTE and UMTS live streaming. When we see the individual CDF of LTE, we observe that about $9 \%$ of the packets are of 54 Bytes, 54-80 Bytes are $26 \%$ of the total, 80-384 Bytes are only 3\%, 384388 Bytes are $8 \%, 388-1374$ are also $8 \%$; while the rest of the packets, about $46 \%$ of the total are of 1374 Bytes.

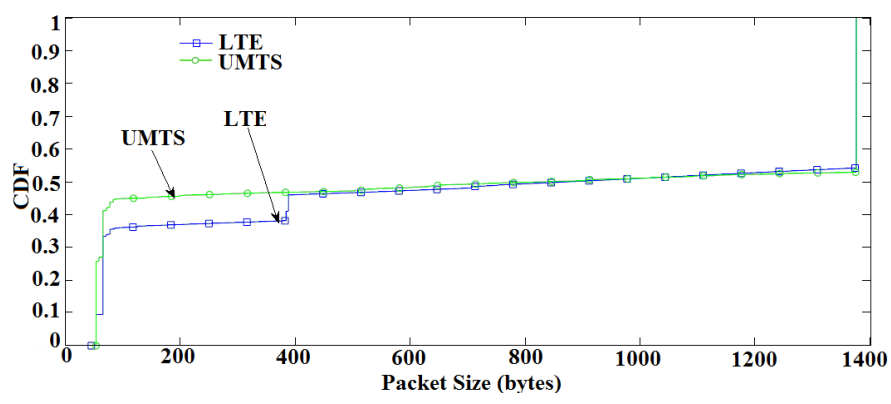

Figure 4: CDF of Packet Sizes for LTE and UMTS Live Streaming

Similarly for the individual CDF of UMTS, we see that about $27 \%$ of packets for UMTS live streaming are of 66 Bytes, 66-91 Bytes are 18\% of the total, 91-1373 Bytes are $8 \%$ of the total; while the rest of the IATs, $47 \%$ of the total use packets of size 1374 Bytes.

When we compare the two wireless communication standards in Figure 4, we notice that for a large part of the communication UMTS uses smaller packet sizes as compared to LTE. Specifically, about $45 \%$ of the packet sizes of UMTS fall below 128 Bytes while that of LTE are of 388 Bytes. Furthermore, there is virtually no difference in the packets sizes used by both LTE and UMTS in live streaming. This can also be verified from Table IV, where the five most frequent packets for both the networks are listed. Although, both LTE and UMTS use packets of size 1374 Bytes most frequently but for the next most frequent packets, the usage is reversed. LTE uses packets of size 66 Bytes more frequently than of 54 Bytes while UMTS do the exact opposite and use packet of size 54 Bytes more frequently than of size 66 Bytes. LTE follows subsequently with larger packets of sizes 388 and 384 Bytes, respectively. Thus, on average LTE is sending more bytes in a single packet.

Table III: Statistics of Live Streaming for LTE \& UMTS IAT

\begin{tabular}{|c|c|c|c|c|c|}
\hline \multicolumn{6}{|c|}{ Statistics of IATs for LTE Live Streaming } \\
\hline$\langle I A T s\rangle(\mathrm{ms})$ & \multicolumn{5}{|c|}{8.5} \\
\hline$\sigma$ of IATs (ms) & \multicolumn{5}{|c|}{45.0} \\
\hline Percentiles & $5 \%$ & $25 \%$ & $50 \%$ & $75 \%$ & $95 \%$ \\
\hline $\log$ (IAT) & -5.30 & -5.22 & -5.05 & -4.54 & -1.36 \\
\hline duration & $5.0 \mathrm{us}$ & 6.0us & $9.0 \mathrm{~ms}$ & 29.0us & 44.0us \\
\hline \multicolumn{6}{|c|}{ Statistics of IATs for UMTS Video Call } \\
\hline$\langle I A T s\rangle(\mathrm{ms})$ & \multicolumn{5}{|c|}{11.3} \\
\hline$\sigma$ of IATs $(\mathrm{ms})$ & \multicolumn{5}{|c|}{48.9} \\
\hline Percentiles & $5 \%$ & $25 \%$ & $50 \%$ & $75 \%$ & $95 \%$ \\
\hline $\log$ (IAT) & -4.82 & -4.39 & -3.04 & -2.28 & -1.24 \\
\hline In seconds & $15.0 \mathrm{us}$ & 40.0us & 904.0us & $5.2 \mathrm{~ms}$ & $58.2 \mathrm{~ms}$ \\
\hline
\end{tabular}

Table IV: Statistics of Live Streaming for Video Call for LTE \& UMTS Packets

\begin{tabular}{|c|c|c|}
\hline \multicolumn{3}{|c|}{ Statistics of Packet Sizes for LTE Live Streaming } \\
\hline Total Packets & \multicolumn{2}{|r|}{1222134} \\
\hline Average Packets/s & \multicolumn{2}{|r|}{116.99} \\
\hline Average throughput & \multicolumn{2}{|r|}{$0.713 \mathrm{Mbps}$} \\
\hline Average Packet size (Bytes) & \multicolumn{2}{|r|}{762.37} \\
\hline \multicolumn{3}{|c|}{ Five Most Frequent Packet Sizes } \\
\hline Packet Sizes (Bytes) & Instances & Relative Frequency (\%) \\
\hline 1374 & 561441 & 45.94 \\
\hline 66 & 290449 & 23.77 \\
\hline 54 & 115865 & 9.48 \\
\hline 388 & 60291 & 4.93 \\
\hline 384 & 35397 & 2.90 \\
\hline \multicolumn{3}{|c|}{ Statistics of Packet Sizes for UMTS Video Call } \\
\hline Total Packets & \multicolumn{2}{|r|}{1306401} \\
\hline Average Packets/s & \multicolumn{2}{|r|}{88.59} \\
\hline Average KBytes/s & \multicolumn{2}{|r|}{64.7} \\
\hline Average Mbps/s & \multicolumn{2}{|r|}{0.52} \\
\hline Average Packet size (Bytes) & \multicolumn{2}{|r|}{730.52} \\
\hline \multicolumn{3}{|c|}{ Five Most Frequent Packet Sizes } \\
\hline Packet Sizes (Bytes) & Instances & Relative Frequency (\%) \\
\hline 1374 & 616976 & 47.23 \\
\hline 54 & 334748 & 25.62 \\
\hline 66 & 185174 & 14.17 \\
\hline 78 & 20153 & 1.54 \\
\hline 59 & 14806 & 1.13 \\
\hline
\end{tabular}

Furthermore, the average packet size of LTE; 762 Bytes as well as the average packets per second 117 packets are much greater than the average packet size of UMTS; 730 Bytes 
and the average packets per second 89 packets. Therefore, the average bits per second of LTE; $0.713 \mathrm{Mbps}$ is also much greater than that of UMTS; $0.52 \mathrm{Mbps}$. The detailed packet details are presented in Table IV. Thus, LTE is not only faster but also more stable than the UMTS network, and performs better for live streaming as well.

\section{CONCLUSION}

Our study on the impact of real time Video with LTE and UMTS revealed that the IAT for LTE is significantly less than that of UMTS, but the packets sizes are larger for LTE. Using such information, one can classify IATs and packet sizes into different sets, or groups, of time assuming a particular Gaussian distribution.

An in-depth peak analysis is going to be the next step of our future work. Since mathematical modeling of the data traffic is effective in analysis of the specific parts of the data in a distributed manner, the knowledge obtained through the process is especially useful for identifying bottlenecks in LTE and UMTS networks. By considering other traffic sources will lead to greater understanding of LTE networks, resulting in LTE networks in providing high quality connections.

\section{ACKNOWLEDGMENT}

Scott Fowler was partially supported by an EC-FP7 Marie Curie CIG grant, Proposal number: 294182. The work leading to these results has also received funding from the European Unions Seventh Framework Programme (FP7/2007-2013) under grant agreement $n^{o}$ [609094 RERUM].

\section{REFERENCES}

[1] K. Hameseder, S. Fowler, and A. Peterson. Performance analysis of ubiquitous web systems for smartphones. In Performance Evaluation of Computer Telecommunication Systems (SPECTS), 2011 International Symposium on, pages 84-89, June 2011.

[2] I. Vidal, I. Soto, M. Calderon, J. Garcia-Reinoso, and V. Sandonis. Transparent network-assisted flow mobility for multimedia applications in ims environments. Communications Magazine, IEEE, 51(7):97-105, July 2013.

[3] R. Trestian, O. Ormond, and G. Muntean. Enhanced power-friendly access network selection strategy for multimedia delivery over heterogeneous wireless networks. Broadcasting, IEEE Transactions on, 60(1):85-101, March 2014.

[4] Cisco. Cisco visual networking index: Global mobile data traffic forecast update, 2013-2018. [ONLINE] http://www.cisco.com/c/en/us /solutions/collateral/service-provider/visual-networking-index-vni/white _paper_c11-520862.pdf, January 2014.

[5] S. Fowler, R.S. Bhamber, and A. Mellouk. Analysis of adjustable and fixed drx mechanism for power saving in lte/lte-advanced. In Communications (ICC), 2012 IEEE International Conference on, pages 1964-1969, June 2012.

[6] R.M. Karthik and A. Chakrapani. Practical algorithm for power efficient drx configuration in next generation mobiles. In INFOCOM, 2013 Proceedings IEEE, pages 1106-1114, April 2013.

[7] Shun-Ren Yang, Sheng-Ying Yan, and Hui-Nien Hung. Modeling umts power saving with bursty packet data traffic. Mobile Computing, IEEE Transactions on, 6(12):1398-1409, Dec 2007.

[8] Jian Liang, Rakesh Kumar, and Keith W. Ross. The kazaa overlay: A measurement study. Computer Networks Journal (Elsevier), 2005.
[9] S.A. Baset and H.G. Schulzrinne. An analysis of the skype peerto-peer internet telephony protocol. In INFOCOM 2006. 25th IEEE International Conference on Computer Communications. Proceedings, pages 1-11, April 2006.

[10] F. Pianese, J. Keller, and E.W. Biersack. Pulse, a flexible p2p live streaming system. In INFOCOM 2006. 25th IEEE International Conference on Computer Communications. Proceedings, pages 1-6, April 2006.

[11] M. Majumder and D. Biswas. Real-time mobile learning using mobile live video streaming. In Information and Communication Technologies (WICT), 2012 World Congress on, pages 469-474, Oct 2012.

[12] K. Mandula, S.R. Meda, and D.K. Jain. Research and implementation of a mobile video streaming application for ubiquitous learning. In Technology Enhanced Education (ICTEE), 2012 IEEE International Conference on, pages 1-6, Jan 2012.

[13] Jin-Hwan Jeong, Yong-Ju Lee, Song-Woo Sok, Hag-Young, and Kimyoo-Hyun Park. Cable-tv-based home video streaming system: practice and experience. Network, IEEE, 23(6):22-28, November 2009.

[14] Chi-Ray Lan, Hsiao-Heng Lu, Chih-Wei Yi, and Chien-Chao Tseng. A p2p hd live video streaming system. In Multimedia Technology (ICMT), 2011 International Conference on, pages 475-478, July 2011.

[15] Maoqiang Song and Jing Zhao. Analysis and design of streaming server of video monitoring system in new generation mobile network. In Wireless Communications, Networking and Mobile Computing (WiCOM), 2011 7th International Conference on, pages 1-5, Sept 2011. 